\title{
Development of Traveling Wave Actuators Using Waveguides of Different Geometrical Forms
}

\author{
Ramutis Bansevicius, ${ }^{1}$ Dalius Mazeika, ${ }^{2}$ Vytautas Jurenas, ${ }^{1}$ \\ Genadijus Kulvietis, ${ }^{2}$ and Vytautas Bakanauskas ${ }^{1}$ \\ ${ }^{1}$ Kaunas University of Technology, Studentu g. 56, LT-51424 Kaunas, Lithuania \\ ${ }^{2}$ Vilnius Gediminas Technical University, Sauletekio al. 11, LT-10223 Vilnius, Lithuania \\ Correspondence should be addressed to Ramutis Bansevicius; ramutis.bansevicius@ktu.lt
}

Received 15 September 2015; Revised 17 December 2015; Accepted 20 December 2015

Academic Editor: Mickaël Lallart

Copyright (C) 2016 Ramutis Bansevicius et al. This is an open access article distributed under the Creative Commons Attribution License, which permits unrestricted use, distribution, and reproduction in any medium, provided the original work is properly cited.

\begin{abstract}
The paper covers the research and development of piezoelectric traveling wave actuators using different types of the waveguides. The introduced piezoelectric actuators can be characterized by specific areas of application, different resolution, and torque. All presented actuators are ultrasonic resonant devices and they were developed to increase amplitudes of the traveling wave oscillations of the contact surface. Three different waveguides are introduced, that is, symmetrical, asymmetrical, and cone type waveguide. A piezoelectric ring with the sectioned electrodes is used to excite traveling wave oscillations for all actuators. Operating principle, electrode pattern, and excitation regimes of piezoelectric actuators are described. A numerical modelling of the actuators was performed to validate the operating principle and to calculate trajectories of the contact points motion. Prototype actuators were made and experimental study was performed. The results of numerical and experimental analysis are discussed.
\end{abstract}

\section{Introduction}

Piezoelectric actuators are widely used for development of nano-/micropositioning and manipulation systems. These actuators are capable of reaching the high resolution and speed of the positioning object and possess many attractive characteristics such as short response time, large output power, compact size, self-braking, and good controllability $[1,2]$. The dimensions of the piezoelectric actuators can vary from millimeters up to tens of centimeters, so they can be used for different industrial applications [3, 4]. Piezoelectric actuators can be excited to vibrate in different vibrational modes such as the extensional vibrational mode, the torsional vibrational mode, the flexural vibrational mode, and the flextensional vibrational mode [5]. In order to increase the output power of the actuator and amplitudes of the vibrations, composite piezoelectric transducers are used. Composite transducers usually consist of piezoceramic elements and metal blocks that are clamped by means of a pretension bolt or are glued [6]. Longitudinal composite transducers named as Langevin transducers are widely used for high power ultrasonic devices. Langevin transducers utilize the $\mathrm{d} 33$ vibration mode of piezoceramics and make piezoceramic parts vibrate under compression stress due to the stress applied by the bolt; therefore mechanical quality factor is increased and the high power conversion efficiency is achieved [6,7]. Different configurations of the boosters and amplification horns are used to increase the amplitudes of longitudinal vibration of the Langevin transducer $[7,8]$.

Another type of composite piezoelectric actuators named as cymbal transducers consists of a piezoelectric ceramic disc and two metal caps which can have different shapes $[9,10]$. They have the advantages of small volume and large displacement amplitude. The radial vibration of the piezoelectric disc is excited and converted into the flexural vibration of the metal caps. The caps serve as mechanical transformers for converting and amplifying the small radial displacement and vibration velocity of the piezoceramic disk 


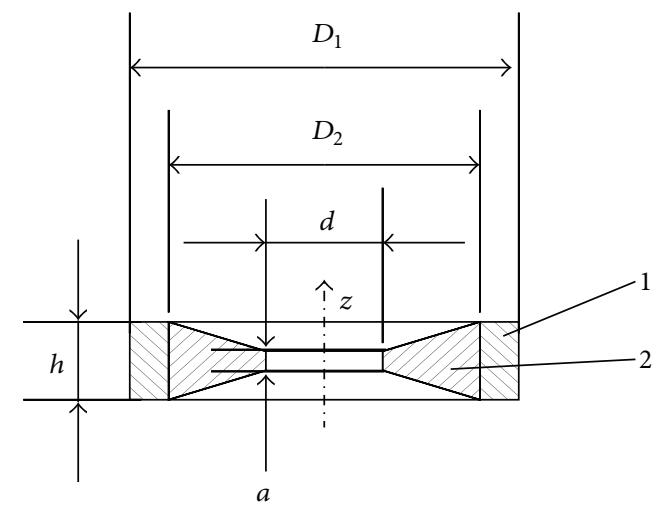

(a)

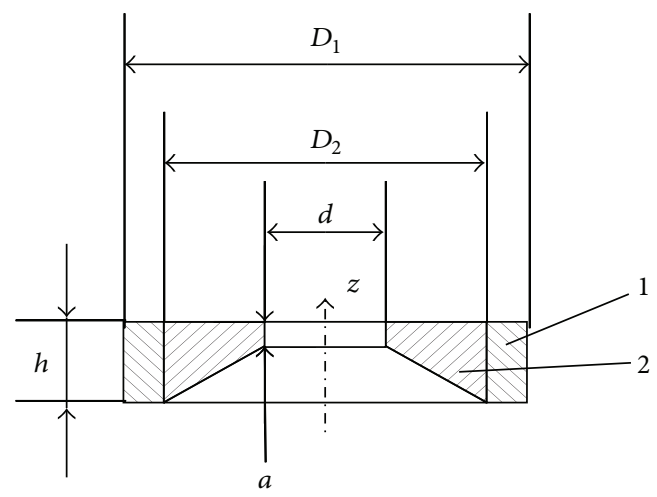

(b)

FIGURE 1: Principle design of the ring type actuators with symmetrical waveguide (a) and asymmetrical waveguide (b); 1, piezoceramic ring, 2, waveguide.

into a much larger axial displacement and vibration velocity normal to the surface of the caps [11].

Traveling wave ultrasonic motors employ traveling wave vibrations of the stator to obtain rotational motion of the rotor. Usually out-of plane bending vibrations of the disc type stator are employed. Several composite type piezoelectric actuators and motors were proposed where in-plane bending and radial vibration mode of the stator is used [12-14]. The output power of the actuators and amplitudes of vibrations are increased because of the metal waveguides of the actuators.

A disc type stator with the teeth is used to improve dynamic characteristics of the contact points, and to obtain better output characteristics of the ultrasonic traveling wave motor $[3,15]$. Vibration amplitudes in circumferential direction and tangent velocity of the contacting points on the stator surface are increased with the increasing tooth height. Therefore rotation speed and the output power of ultrasonic motor are increased as well.

Three new designs of the traveling wave actuators with the special waveguides are proposed and analyzed in this paper. Vibration amplitudes of radial, circumferential, and axial directions are increased because of a specific design of the waveguides. The operating principle of the actuators is described and the results of numerical investigation based on finite element modeling are given. Prototype actuators were fabricated and corresponding measurements were done. Finally, the results of numerical simulation and experimental study are analyzed and discussed.

\section{Design and Operating Principle}

All proposed piezoelectric actuators are composite type ultrasonic devices and are used for the precise rotation of a positioning object such as a sphere and a cone. A positioning object must be located in the center of the actuator. All actuators consist of a piezoelectric ring and a metal waveguide. Electrodes of the piezoelectric ring are divided into several sections. A traveling wave of the outof-plane or in-plane vibrations is formed in the ring by the superposition of two vibration mode responses with the equal amplitude and the phase difference in both time and space. Traveling wave vibrations of the piezoelectric ring are transferred to the waveguide so the vibration amplitudes of the contacting points located at the surface of the internal circle of the waveguide are increased.

The principle design piezoelectric actuators with the ring type waveguides are shown in Figure 1. Actuators consist of the two parts: a piezoelectric ring and an inner metal ring. Both parts are glued using epoxy resin. An inner metal ring is a waveguide of the actuator. The waveguide is made from aluminum. The outer and inner diameters of the piezoceramic ring are denoted as $D_{1}$ and $D_{2}$, respectively. The thickness of the external and internal circles of the waveguide is denoted as $h$ and $a$ (Figure 1). The piezoelectric ring is polarized in the thickness direction.

Two different configurations of the waveguides are proposed, that is, a ring with symmetrical and asymmetrical cross sections (Figure 1). Both waveguides have variable thickness in the radial direction. The ring thickness decreases toward the center of the ring; therefore vibration amplitudes are amplified. The difference between two proposed waveguides is a cross-section shape, that is, symmetrical and asymmetrical. The symmetric waveguide amplifies vibrations of the piezoceramic ring but does not change its direction while the asymmetric waveguide amplifies and decomposes vibrations into the axial and radial components; therefore the axial force and accordingly normal force between contacting surfaces of the rotor and waveguide is increased. So the asymmetric waveguide can be used for high power applications while the symmetric waveguide enables achieving a large rotation speed of the rotor.

Two different electrode topologies are used to excite traveling wave vibrations. The first electrode topology has six equal sectors which are grouped into three groups (Figure 2(a)). Three phases of the voltages shifted by $2 / 3 \pi$ are applied on the corresponding groups of the electrodes. 


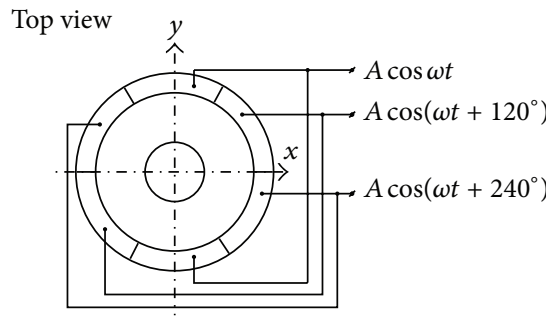

(a)

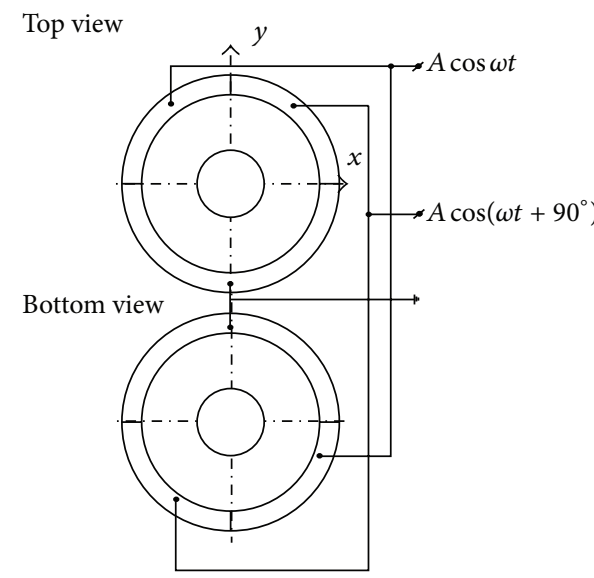

(b)

Figure 2: Electrode topologies and excitation schemes: six electrodes and three electric signals (a); six electrodes and two electric signals (b).

The bottom electrode is grounded. The second topology has six electrodes as well but only two electric signals with the phase shifted by $\pi / 2$ are used (Figure $2(b)$ ). The electrodes are located at the top and bottom surfaces of the ring and are divided into three sectors. Two sectors have the angle size equal to $\pi / 2$ while the size of the third sector is equal to $\pi$. Electrodes with the $\pi / 2$ angle are used for the excitation. Electrode sectors with the angle equal to $\pi$ are grounded. The advantage of this electrode topology is that only two electric signals are used for the excitation.

Two different bending modes can be employed to rotate rotor, that is, $\mathrm{B}_{12}$ out-of-plane bending mode and $\mathrm{B}_{02}$ inplane bending mode. Traveling waves vibrations are actuated in the internal circle of the waveguide when aforementioned excitation regimes of the electrodes are used. Two outof-plane travelling waves are actuated when $\mathrm{B}_{12}$ out-ofplane mode is used. If the rotor is located in the center of the waveguide and prepressured to the top surface, then the vibration with microamplitude of points on waveguide surface will be transformed to a rotary motion of the rotor through frictional force. The rotation of the rotor also will be obtained when $\mathrm{B}_{02}$ in-plane bending mode is actuated. In this case two in-plane traveling wave vibrations of the waveguide rotate rotor through the frictional forces.

The main advantage of the proposed waveguides is that they can operate in two resonant modes. Amplitudes of traveling wave vibrations are amplified in the radial and circumferential directions when the in-plane vibration mode is used. If out-of plain vibrations are excited, then the vibrations in axial direction of the waveguide are amplified.

Figure 3 shows a piezoelectric travelling wave actuator with a hollowed cone waveguide. A piezoceramic ring is glued up at the bottom of the waveguide. Electrodes of the piezoceramic ring are divided into four equal sections. Four electric signals with the phases shifted by $\pi / 2$ are used to excite out-of-plane travelling wave vibrations of the piezoceramic ring. These vibrations are transferred into the axial traveling wave vibrations of the cone. Amplitudes of the vibrations in axial direction are magnified at the top surface of the cone due to the difference of the cross-sections area at the top and bottom of the cone. The top surface of the cone is used as a contacting surface to rotate positioning object about the $z$ axis.

\section{Numerical Simulation}

Finite element modeling was used to validate the operation principle of the proposed traveling wave actuators and to analyze the dependence of amplification ratio from dimensions of the waveguides. Numerical study included modal frequency analysis and harmonic response analysis. FEM software ANSYS was used to build a three-dimensional finite element model of the actuators and to run a simulation. The finite element models contain all components of the actuators mentioned in the previous section. The following materials were used in the finite element model: PZT- 8 was used for modeling piezoceramic rings, and aluminium AW6082 was used for the waveguides. The glue layer was neglected in the model. The properties of the materials used to build a finite element model are given in Table 1.

The dimensions of the ring type and cone type traveling wave actuators are given in Table 2. The thickness of the internal circle of the ring initially was set to $2 \mathrm{~mm}$ for both ring type actuators. Height $h$ was equal to $57 \mathrm{~mm}$ for the cone type waveguide.

Actuators were not clamped in the models. Damping was evaluated by introducing the mechanical loss factor for piezoceramic rings and the isotropic loss factor of the metallic parts. The mechanical loss factor was set to 0.004 for the piezoceramic rings and the isotropic loss factor equal to 0.001 was set for the aluminium waveguide. Piezoelectric and dielectric losses were neglected in the model.

Modal-frequency analysis of the actuators was performed. Short circuit type electric boundary conditions were applied; that is, a constant voltage of zero was applied at the electrodes of piezoelectric ring. While analyzing the 


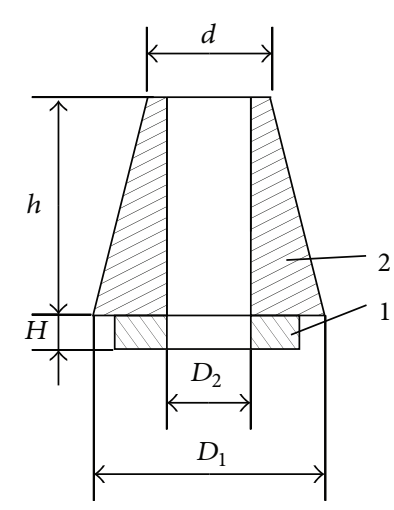

(a)

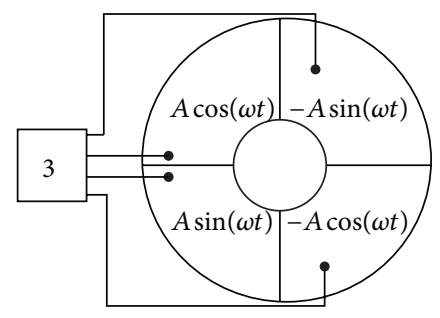

(b)

FIGURE 3: Principle scheme of the cone type actuators (a) and the electrodes topology (b): 1, piezoceramic ring, 2, cone type waveguide, and 3 , signal generator.

TABLE 1: Properties of the actuator materials.

\begin{tabular}{lc}
\hline Material properties & Piezoceramic PZT-8 \\
\hline Young's modulus $\left[\mathrm{N} / \mathrm{m}^{2}\right]$ & \\
\hline Poison's ratio & 7700 \\
\hline Density $\left[\mathrm{kg} / \mathrm{m}^{3}\right]$ & $\varepsilon_{11}=11.42 ; \varepsilon_{33}=8.85$ \\
\hline Permittivity, $\times 10^{-7}[\mathrm{~F} / \mathrm{m}]$ & $e_{13}=-18.01 ; e_{33}=29.48 ; e_{52}=10.34$ \\
\hline Piezoelectric matrix $\left[\mathrm{C} / \mathrm{m}^{2}\right]$ & $c_{11}=14.68 ; c_{12}=8.108 ; c_{13}=8.105 ; c_{33}=13.17 ; c_{44}=3.29 ; c_{66}=3.14$ \\
\hline Elasticity matrix, $\times 10^{10}\left[\mathrm{~N} / \mathrm{m}^{2}\right]$ & 2700 \\
\hline
\end{tabular}

TABLE 2: Dimensions of the actuators.

\begin{tabular}{lccc}
\hline Dimensions & $\begin{array}{c}\text { Symmetrical } \\
\text { actuator }\end{array}$ & $\begin{array}{c}\text { Asymmetrical } \\
\text { actuator }\end{array}$ & $\begin{array}{c}\text { Cone type } \\
\text { actuator }\end{array}$ \\
\hline$D_{1}(\mathrm{~mm})$ & 40 & 40 & 57 \\
$D_{2}(\mathrm{~mm})$ & 32 & 32 & 36 \\
$d(\mathrm{~mm})$ & 10 & 10 & 32 \\
$H(\mathrm{~mm})$ & 5 & 5 & 5 \\
\hline
\end{tabular}

results of oscillation modes of the ring type actuators, $\mathrm{B}_{12}$ modal shape of out-of-plane bending vibrations was obtained at the frequency $56.82 \mathrm{kHz}$ and $56.71 \mathrm{kHz}$ for symmetrical and asymmetric actuator, respectively (Figure 4(a)). The edges of the internal circle of the waveguide can be used as contact surface to drive a rotor when traveling wave excitations signals are applied on the electrodes. This mode will be investigated in more detail. $\mathrm{B}_{02}$ out-of plane vibration mode was obtained at $72.22 \mathrm{kHz}$ for both actuators. The first expansion mode in the axial direction of the cone type waveguide is obtained at frequency $45.63 \mathrm{kHz}$ and will be used as operating mode of the actuator (Figure 4(b)).

Harmonic response analysis was performed with the aims to find out the response of the actuators to sinusoidal voltage and to find the trajectories of the contact points' motion.
The excitation schemes shown in Figures 2(a) and 3(b) were used for numerical simulations. A $100 \mathrm{~V}$ AC signal was applied to the electrodes. A frequency range from $40 \mathrm{kHz}$ to $60 \mathrm{kHz}$ with a solution of $100 \mathrm{~Hz}$ intervals was studied and the vibration amplitudes of the contact points located on the top edge of internal circle of the waveguides were analyzed. Adequate response curves of the contact point oscillation amplitudes are given in Figures 5 and 6.

The graphs of the contact point vibration amplitude in the axial direction $u_{z}$ show the local peaks at $56.7 \mathrm{kHz}$ and $56.6 \mathrm{kHz}$ for the symmetric and asymmetric actuators, respectively. These resonant frequencies correspond to $B_{12}$ vibration mode. It also can be noticed that the amplitude of vibrations in the axial direction $u_{z}$ of the symmetrical waveguide is 3.9 times larger than that of the asymmetrical waveguide. It can be explained because of a smaller bending stiffness of the symmetric actuator. The amplitude-frequency characteristic of the contact point of cone type actuator has several local peaks because different vibration modes are excited in the analyzed frequency range. The largest peak of vibration component $u_{z}$ is obtained at $45.6 \mathrm{kHz}$. The vibration mode presented in Figure 4(b) is excited at this resonant frequency.

The trajectories of the contact point motion were calculated (Figure 7). By observing elliptical trajectories it can be noticed that the contact point motion trajectory of 


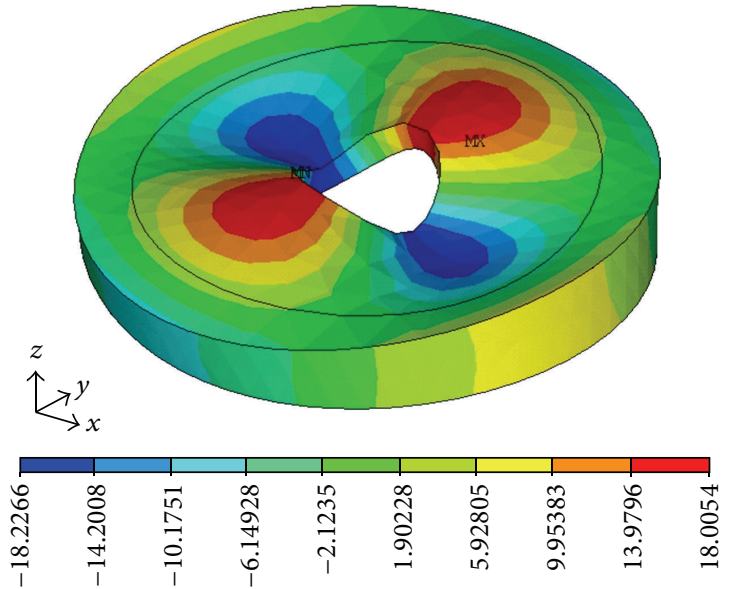

(a)

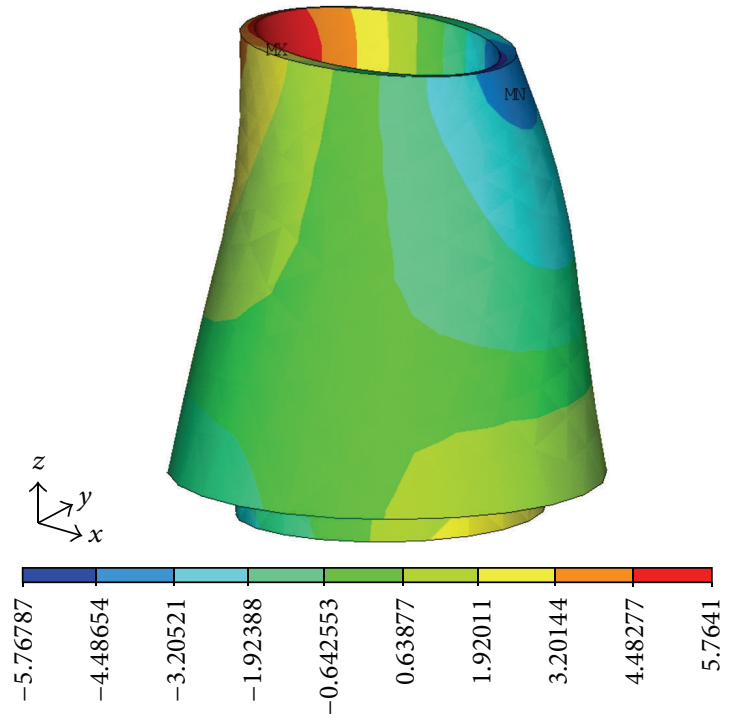

(b)

FIGURE 4: Modal shapes of the analyzed actuators: symmetrical ring type (a), cone type (b).

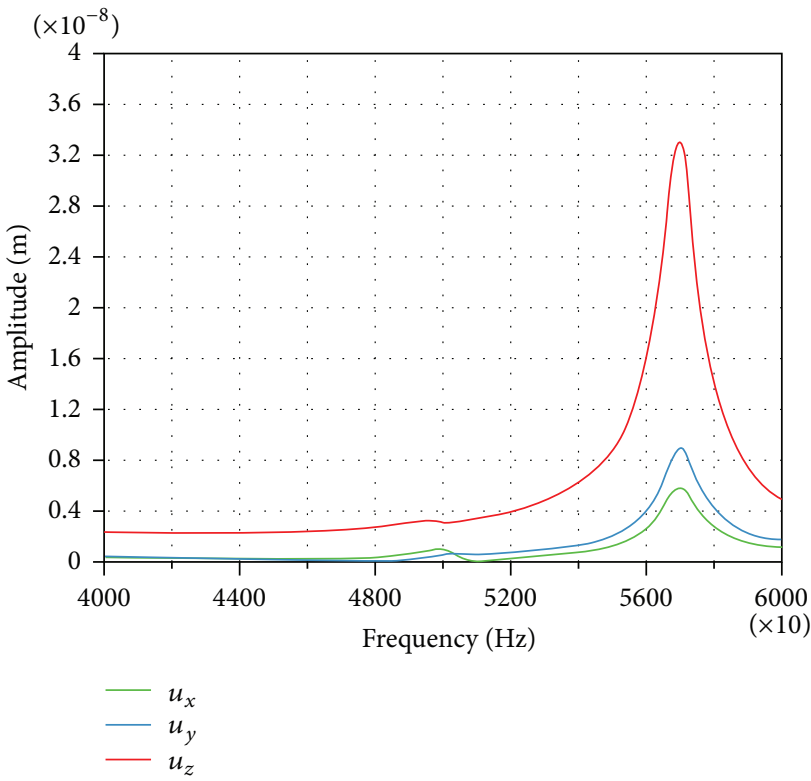

(a)

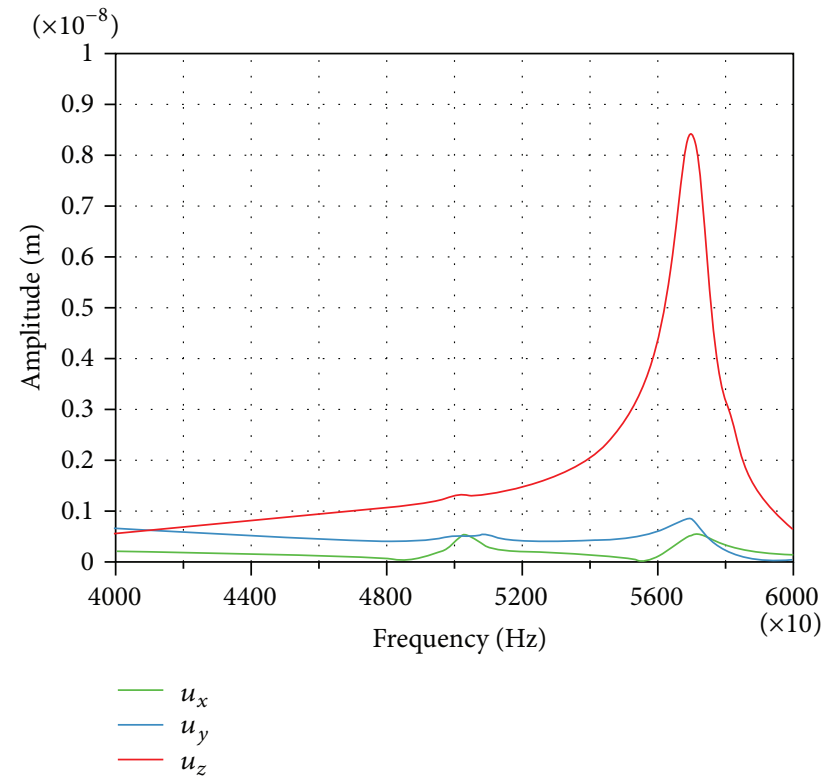

(b)

FIGURE 5: The amplitude-frequency characteristic of the contact point oscillation at three-phase excitation with $A=100 \mathrm{~V}$ : symmetrical waveguide (a), asymmetrical waveguide (b).

the symmetrical ring type actuator has length of the major axis 4.0 times larger than that of the asymmetrical actuator. It means that the velocity of the contact point motion is higher and the impact is much more powerful.

The dependence of the major semiaxis length of the contact point elliptical motion on the thickness ratio $a / h$ of the ring type waveguide internal and external circles was analyzed (Figure 8(a)). The graphs show that length of the major semiaxes has a nonlinear dependence on the thickness of the internal circle for both waveguides. Vibration amplitudes and length of the major semiaxis decrease when thickness of the internal circle increases in case of symmetrical waveguide. The length of the major semiaxis of asymmetric waveguide has local minimum when the ratio between the external and internal thickness of the ring is equal to 0.6 and increases when the thickness is smaller or larger than $3 \mathrm{~mm}$. 


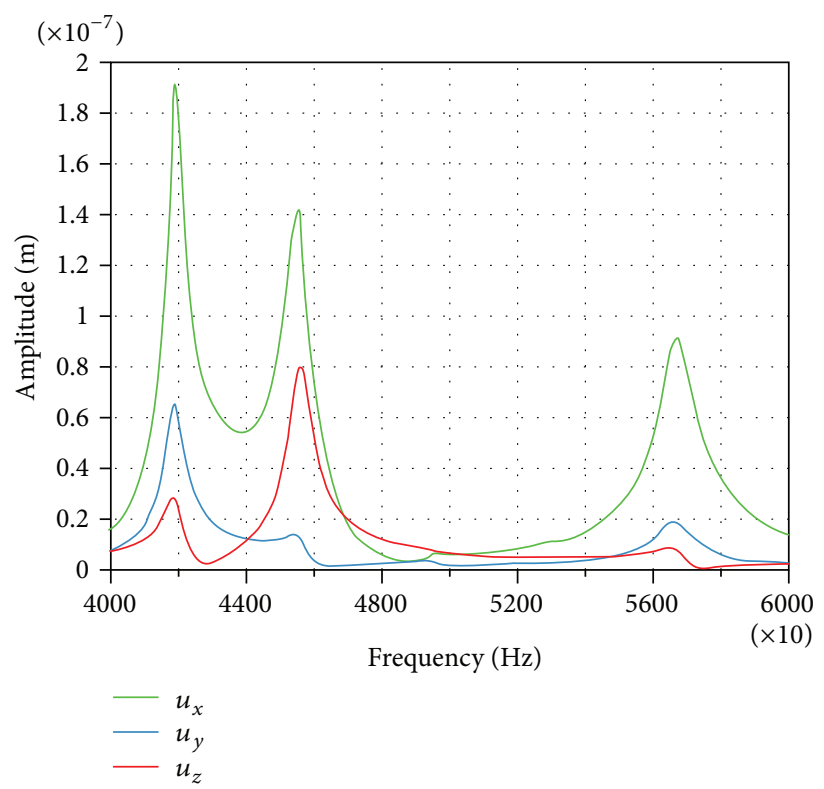

FIGURE 6: The amplitude-frequency characteristic of the contact point oscillation.

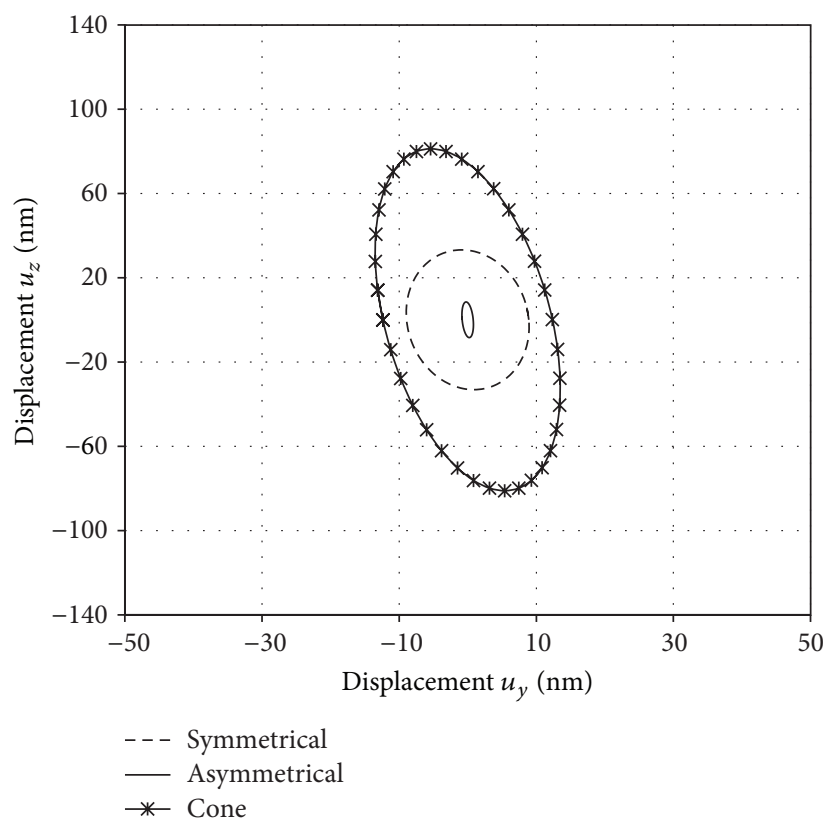

FIGURE 7: Trajectories of the contact point motion of analyzed piezoelectric actuators.

This means that the shape of an asymmetric cross-section of the waveguide has a strong influence on the amplification of vibration amplitudes of the contact points.

The dependence of the major semiaxis length of the contact point elliptical motion on the ratio $h / D_{1}$ of the cone type waveguide is shown in Figure 8(b). It can be noticed that the dependence is nonlinear and the largest length of ellipses semiaxis is achieved when the ratio value is equal to 0.8 . Special cases of cone type waveguide design can be analyzed, that is, when the first longitudinal mode coincides with the first expansion mode of the cone. In this case the largest amplitudes of contact surface vibrations will be achieved. However contact time between surface of the rotor and stator will be reduced at least by a half of the period of longitudinal vibrations in this case.

\section{Experimental Investigation}

Prototype piezoelectric actuators with symmetrical, asymmetrical, and cone type waveguides were fabricated (Figure 9). The goal of an experimental investigation 


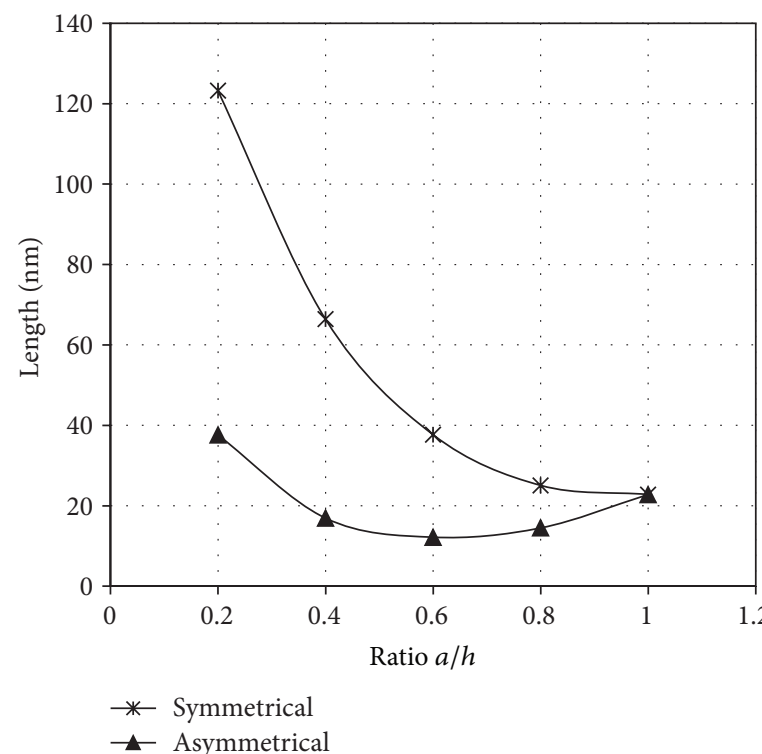

(a)

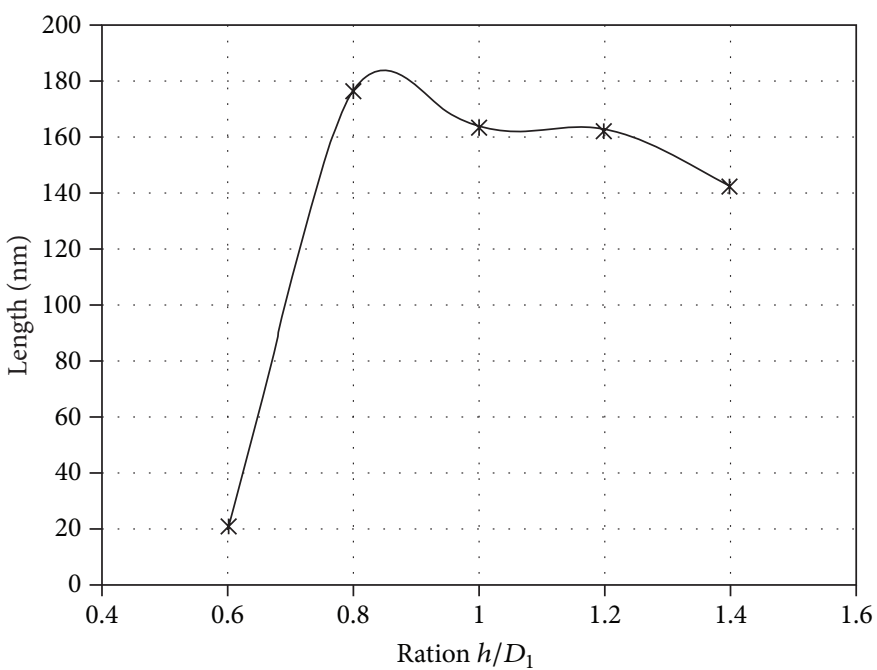

(b)

FIGURE 8: The dependence of the length of ellipses major semiaxis on thickness ratio of the ring type waveguide (a); height of the cone (b).

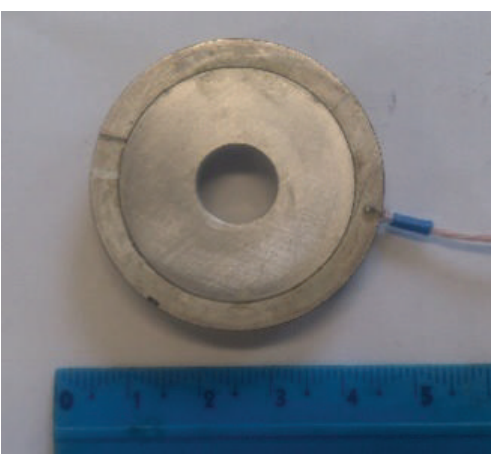

(a)

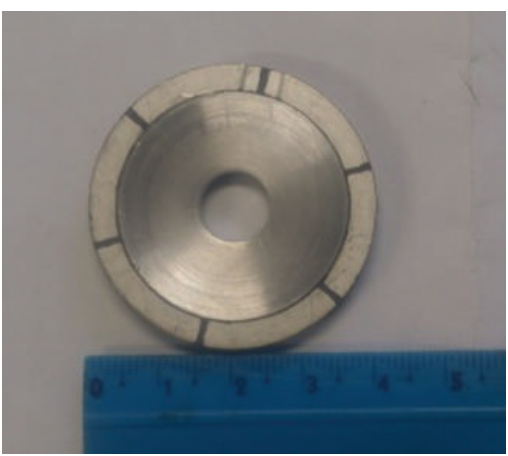

(b)

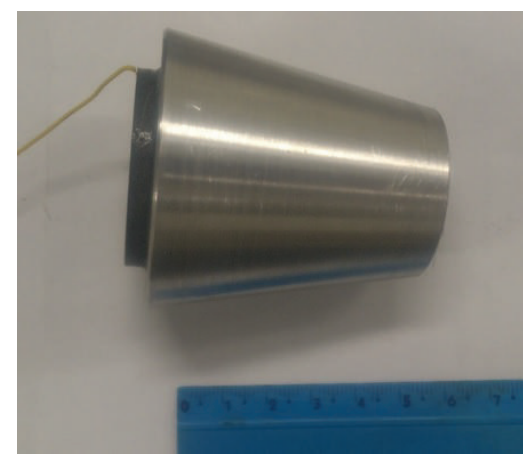

(c)

FIGURE 9: Prototype travelling wave actuators: ring type actuator with asymmetrical waveguide (a), ring type actuator with asymmetrical waveguide (b), and cone type actuator (c).
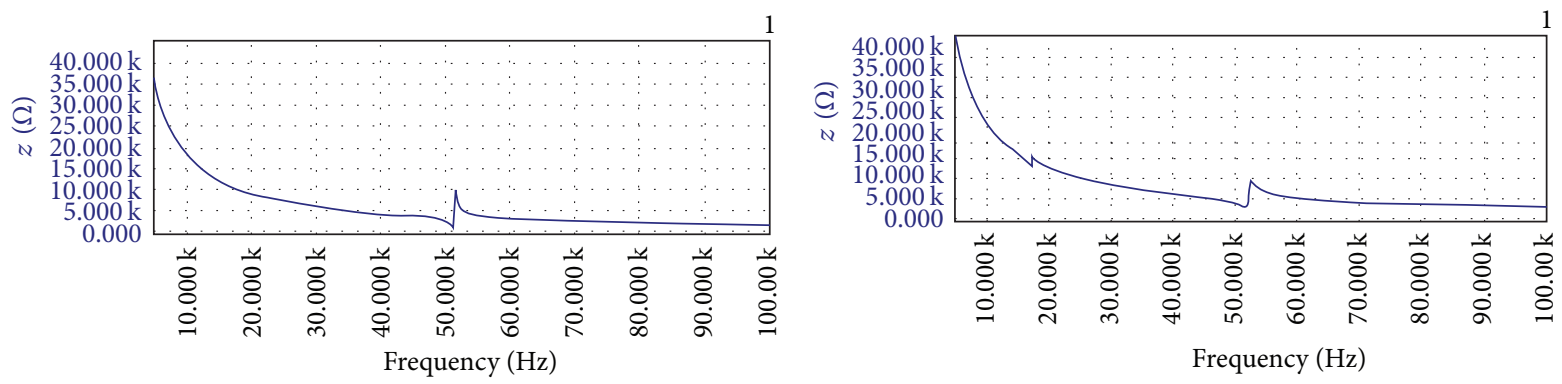

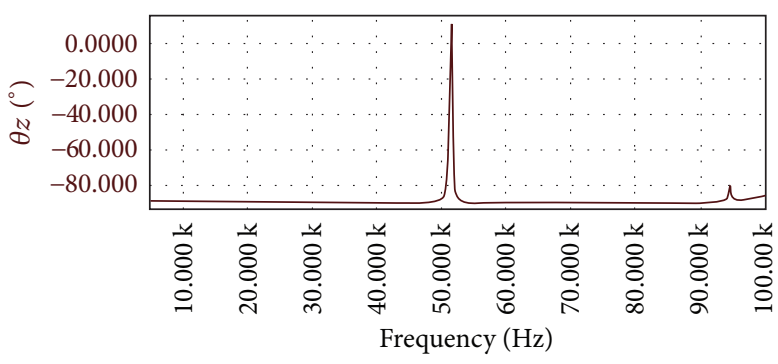

(a)

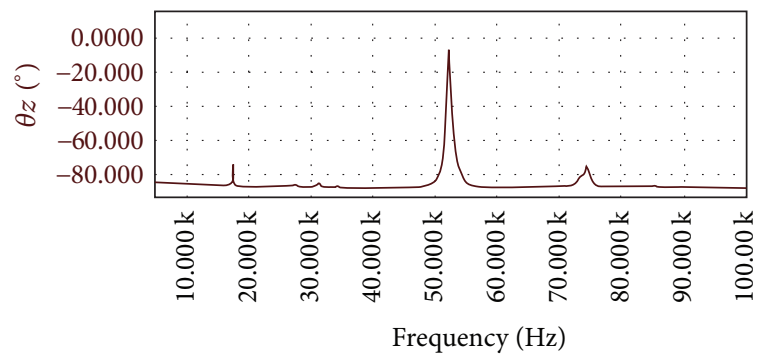

(b)

FIGURE 10: Measured impedance and phase of the symmetrical (a) and asymmetric (b) ring type actuator versus frequency. 


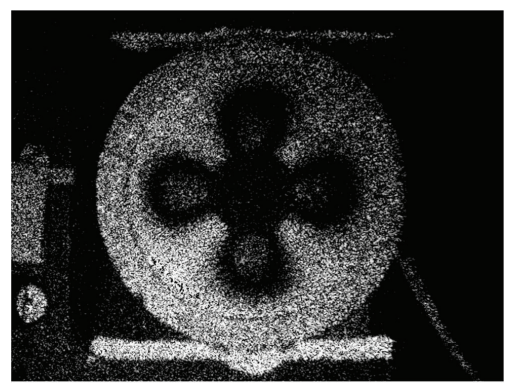

FIGURE 11: Hologram of $\mathrm{B}_{12}$ modal shape of the actuator with asymmetric waveguide $(53.2 \mathrm{kHz})$.
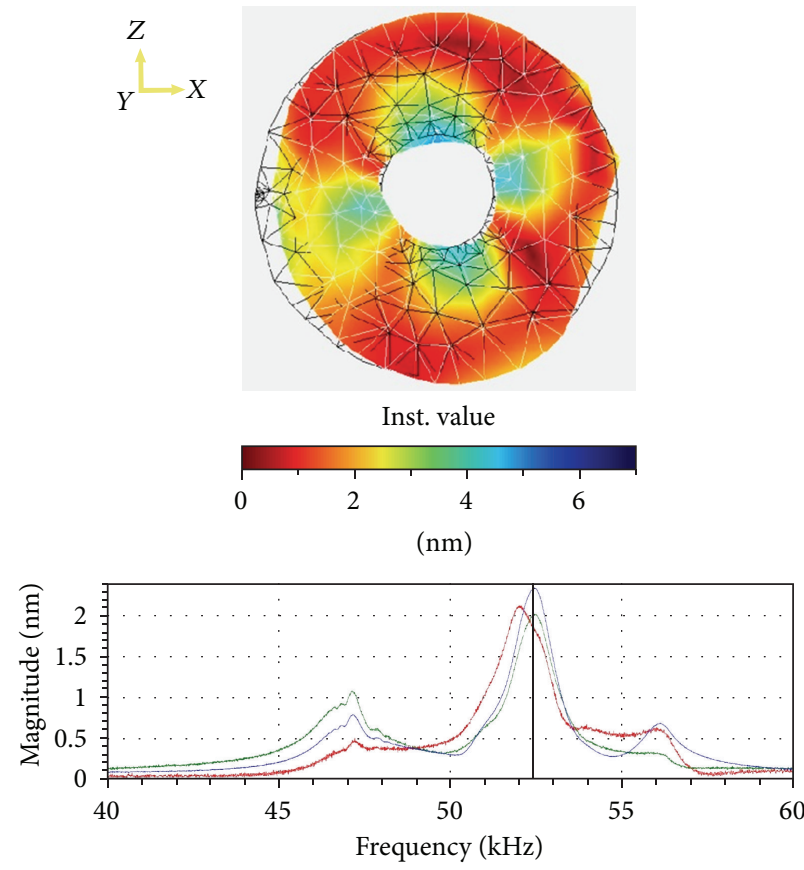

Frequency $52.445 \mathrm{kHz}$

- Magnitude $1.7887 \mathrm{~nm}$

- Magnitude $1.9928 \mathrm{~nm}$

- Magnitude $2.3184 \mathrm{~nm}$

(a)
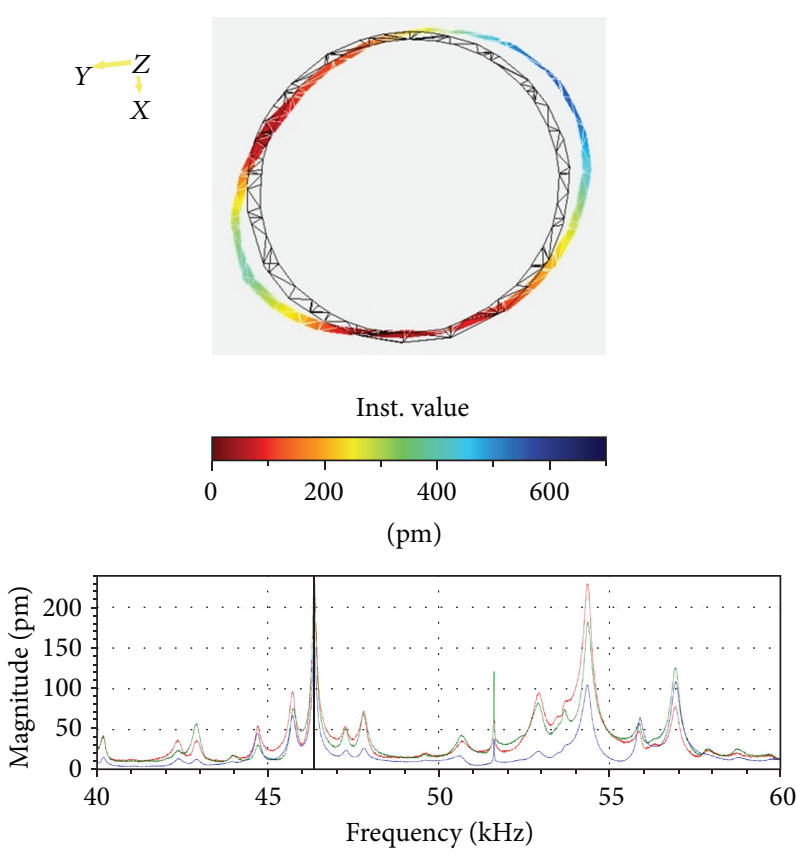

Frequency $46.367 \mathrm{kHz}$

- Magnitude $232.41 \mathrm{pm}$

- Magnitude $225.44 \mathrm{pm}$

- Magnitude $151.13 \mathrm{pm}$

(b)

FIGURE 12: Distribution of displacement amplitudes on the top surface of actuators: symmetric ring type actuator at $52.445 \mathrm{kHz}$ (a), cone type actuator $(46.367 \mathrm{kHz})(\mathrm{b})$.

was to validate operating principle of the actuator and to verify results of numerical modelling. Impedancefrequency characteristics of the ring type symmetrical and asymmetrical actuator were measured with the help of the impedance analyzer Wayne Kerr 6500B. The results of the measurements are given in Figure 10. The curve valley position at $52.445 \mathrm{kHz}$ indicates $\mathrm{B}_{12}$ resonant mode of outof-plane vibrations of symmetrical waveguide (Figure 10(a)) and curve valley position at $53.2 \mathrm{kHz}$ indicates the same resonant mode of actuator with asymmetrical waveguide. The relative errors between the measured and calculated resonant frequency are $7.5 \%$ and $6.0 \%$, respectively, for symmetric and asymmetric actuator. The errors mainly came from FEM simulation, such as the inaccuracy of the material property parameters between the real and the simulated ones, having neglected glue layer.

The hologram of $\mathrm{B}_{12}$ resonant mode of the actuator is shown in Figure 11. It can be noticed that the shape of the resonant mode fully corresponds to the modal shape obtained during numerical simulation.

The top surface's oscillations of the ring type actuator with symmetric waveguide and cone type actuator were measured using a vibrometer POLYTEC PSV 500. The resonant frequency at $52.445 \mathrm{kHz}$ was determined for the symmetric ring type actuator (Figure 12(a)). The distribution of oscillation amplitudes on the top surface of the actuator is the same as was obtained in numerical simulation. Distribution of the oscillation amplitudes on top surface of the cone type actuator 
at $46.367 \mathrm{kHz}$ is shown in Figure 12(b). It can be noticed that the distribution is similar as it was calculated. Difference between calculated and measured resonant frequencies is $1.65 \%$. The operating principle of all three actuators also were validated by studying rotation of the steel ball located at the center of the actuator. It confirms that all actuators can be used for rotation of the positioning objects or rotational type motors.

\section{Conclusions}

A novel design of the three waveguides used for the traveling wave actuators was proposed. Numerical and experimental investigations confirm the possibility of achieving elliptical trajectories of contacting surfaces and rotating the rotor using different excitation schemes of the piezoelectric actuators. The length of the major semiaxis has a nonlinear dependence on the thickness of the internal circle of the asymmetrical waveguide and the height of the external radius ratio of the cone type waveguide. The length of the major semiaxis of contact point vibrations increases when the thickness of the internal circle of the symmetrical ring type waveguide decreases. Values of the resonant frequency from the finite element model are in good agreement with the results from the experimental investigation.

\section{Conflict of Interests}

The authors declare that there is no conflict of interests regarding the publication of this paper.

\section{Acknowledgment}

This work was supported by the Research Council of Lithuania under Project No. MIP-045/2014.

\section{References}

[1] K. Uchino, Piezoelectric Actuators and Ultrasonic Motors, Kluwer Academic Publishers, Boston, Mass, USA, 1997.

[2] T. Hemsel and J. Wallaschek, "Survey of the present state of the art of piezoelectric linear motors," Ultrasonics, vol. 38, no. 1, pp. 37-40, 2000.

[3] Ch. Zhao, Ultrasonic Motors: Technologies and Applications, Springer, Berlin, Germany; Science Press, Beijing, China, 2011.

[4] T. Morita, "Miniature piezoelectric motors," Sensors and Actuators A: Physical, vol. 103, no. 3, pp. 291-300, 2003.

[5] O. E. Mattiat, Ultrasonic Transducer Materials, Springer, New York, NY, USA, 1971.

[6] S. Ueha, Y. Tomikawa, M. Kurosawa, and N. Nakamura, Ultrasonic Motors. Theory and Applications, Clarendon Press, Oxford, UK, 1993.

[7] K. Asumi, R. Fukunaga, T. Fujimura, and M. K. Kurosawa, "High speed, high resolution ultrasonic linear motor using Vshape two bolt-clamped Langevin-type transducers," Acoustical Science and Technology, vol. 30, no. 3, pp. 180-186, 2009.

[8] R. Bansevicius, R. Barauskas, G. Kulvietis, and K. Ragulskis, Vibromotors for Precision Microrobots, Hemisphere Publishing Corporation, Washington, DC, USA, 1988.
[9] J. Zhang, W. J. Hughes, P. Bouchilloux, R. J. Meyer Jr., K. Uchino, and R. E. Newnham, "A class V flextensional transducer: the cymbal," Ultrasonics, vol. 37, no. 6, pp. 387-393, 1999.

[10] R. J. Meyer Jr., A. Dogan, C. Yoon, S. M. Pilgrim, and R. E. Newnham, "Displacement amplification of electroactive materials using the cymbal flextensional transducer," Sensors and Actuators A: Physical, vol. 87, no. 3, pp. 157-162, 2001.

[11] C.-L. Sun, S. S. Guo, W. P. Li, Z. B. Xing, G. C. Liu, and X.Z. Zhao, "Displacement amplification and resonance characteristics of the cymbal transducers," Sensors and Actuators A: Physical, vol. 121, no. 1, pp. 213-220, 2005.

[12] T. Zhou, Y. Chen, D. Fu, X. Hu, Y. Li, and B. Tian, "Integrated lens auto-focus system driven by a nut-type ultrasonic motor (USM)," Science in China Series E: Technological Sciences, vol. 52, no. 9, pp. 2591-2596, 2009.

[13] W. Wischnewskiy, "Piezoelectric motor," US Patent no. US5872418 A, 1999.

[14] S. Lin, "Study on the radial vibration of a piezoelectric ceramic thin ring with an inner metal disc," Journal of Physics D: Applied Physics, vol. 39, no. 21, 2006.

[15] Y. Liu, W. Chen, J. Liu, and S. Shi, "A cylindrical traveling wave ultrasonic motor using longitudinal and bending composite transducer," Sensors and Actuators A: Physical, vol. 161, no. 1-2, pp. 158-163, 2010. 


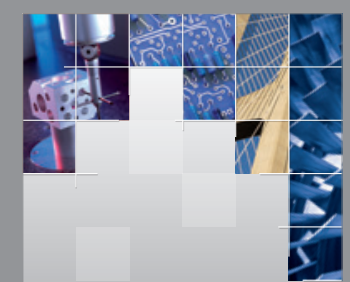

\section{Enfincering}
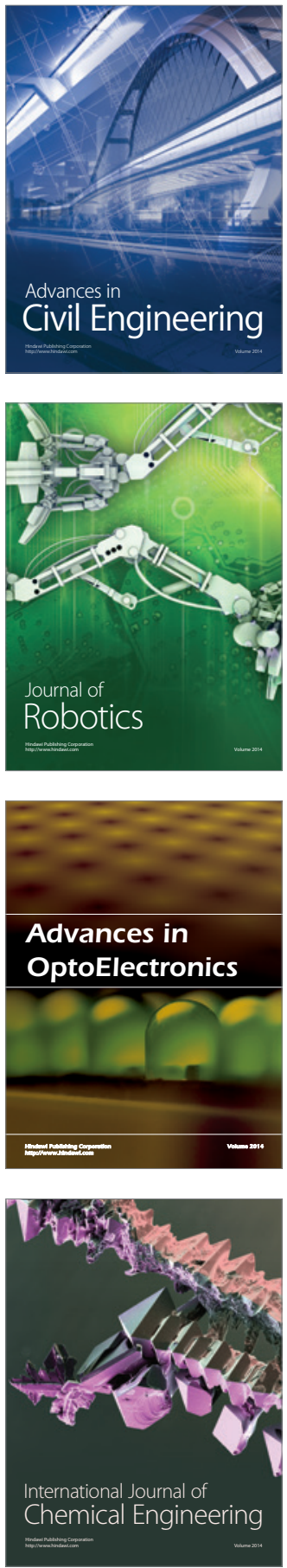

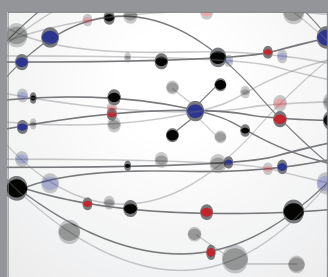

The Scientific World Journal

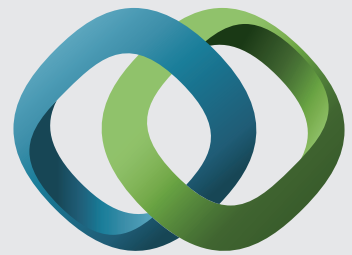

\section{Hindawi}

Submit your manuscripts at

http://www.hindawi.com
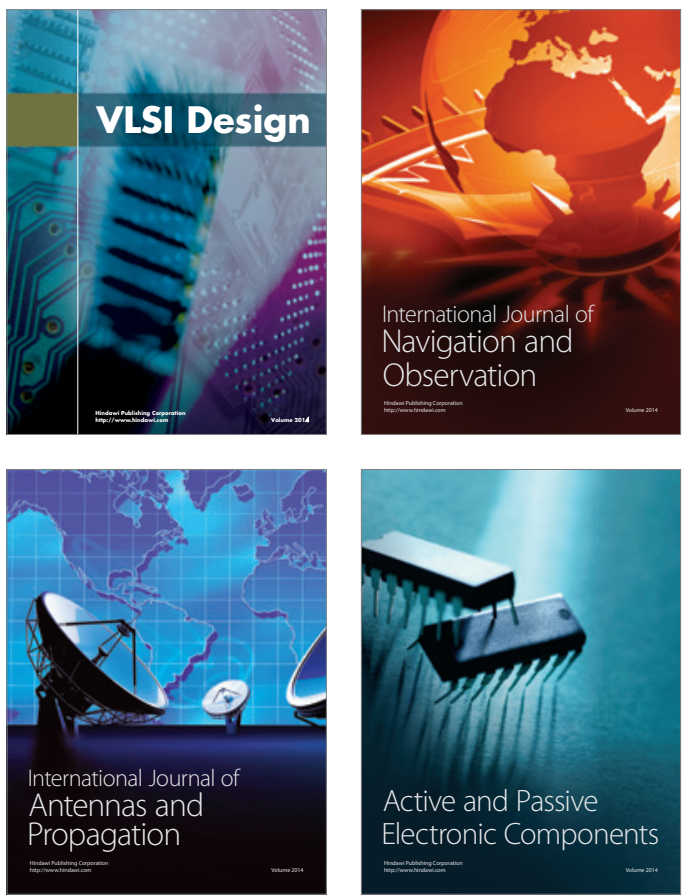
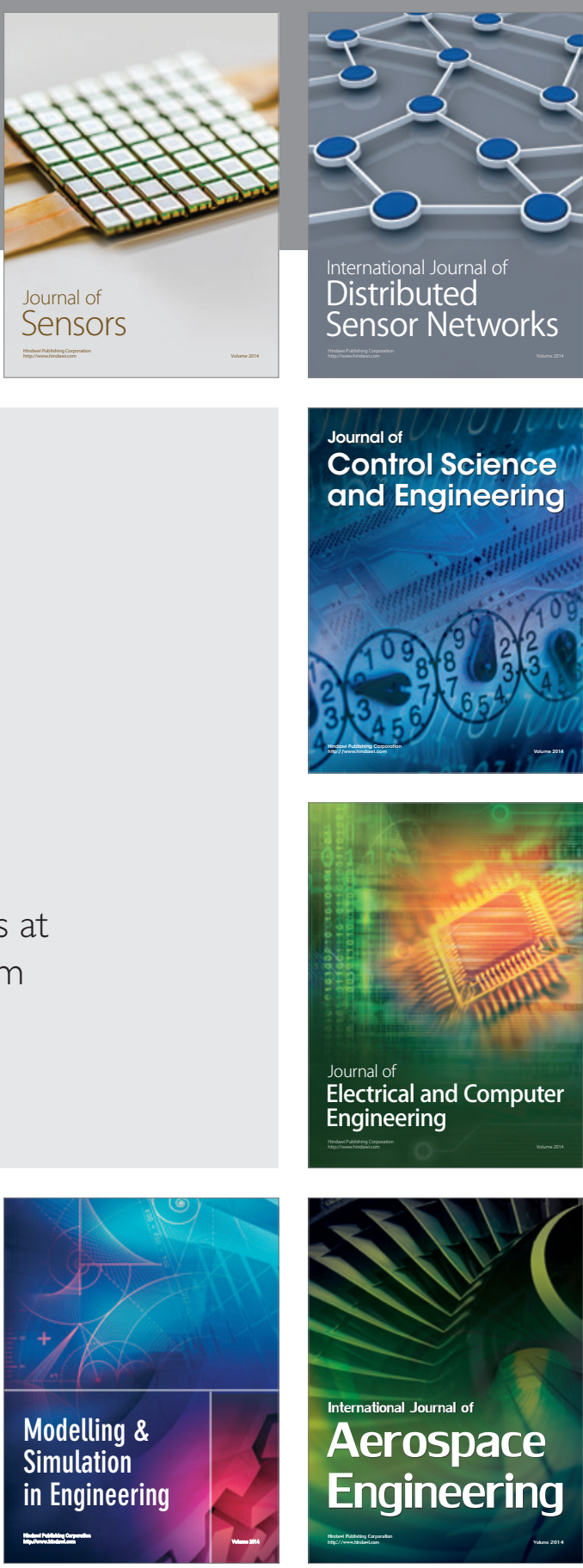

International Journal of

Distributed

Sensor Networks

Journal of

Control Science

and Engineering
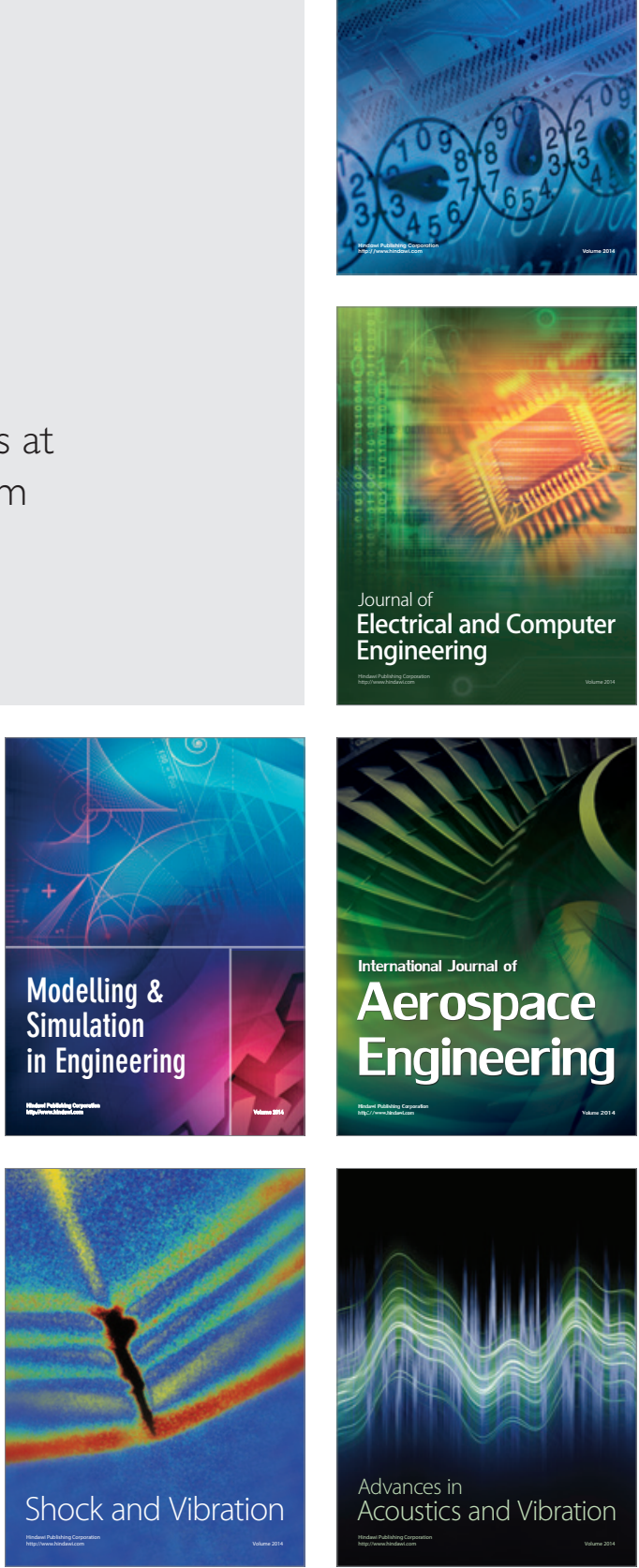